\title{
Microvascular Anatomy of Extrahepatic Bile Ducts in Adult South African Clawed Toad, Xenopus laevis Daudin: Scanning Electron Microscopy of Vascular Corrosion Casts and Correlative Light Microscopy
}

\author{
A. Lametschwandtner ${ }^{*}$, U. Lametschwandtner ${ }^{*}$, H. Bartel ${ }^{*}$, C. Radner ${ }^{*}$ and B. Minnich ${ }^{*}$ \\ *University of Salzburg, Department of Organismic Biology, Vascular and Muscle Research Unit, \\ Hellbrunnerstrasse 34, A-4020 Salzburg, Austria,
}

While the microvascular anatomy of extrahepatic ducts in mammals is studied by scanning electron microscopy (SEM) of vascular corrosion casts and was found to consist of an outer serosal and an inner subepithelial vascular plexus [1-6] the microvasculature of extrahepatic bile ducts in lower vertebrates is mentioned in a brief account only [7]. The present study assumes that in the carnivorous Xenopus the microvascular bed of the extrahepatic bile ducts is similar to that in mammals to serve the same functions, i.e. nourishment of the ducts walls and exchange of water and electrolytes between bile and peribiliary blood.

Six female and two male adult Xenopus laevis (body weights ranging from 48g-92 g) were cast via the ventricle - conus arteriosus with the polymerizing resin Mercox-Cl-2B (Ladd Res. Inc., Burlington, VT), diluted $4+1(\mathrm{v}+\mathrm{v})$ with monomeric methyl-methacrylic acid (Fluka Chemicals, Basle, $\mathrm{CH})$ using manual pressure. Injected animals were macerated $\left(7,5 \% \mathrm{KOH} ; 40^{\circ} \mathrm{C}, 12-24 \mathrm{~h}\right)$, rinsed and frozen in distilled water, freeze-dried, mounted onto specimen stubs, evaporated with carbon and gold, and examined in an SEM (Cambridge 250, UK) at $10 \mathrm{kV}$. For light microscopy two animals were fixed with Bouin's solution by vascular perfusion, extrahepatic bile ducts were removed, immersed in fresh fixative, rinsed, dehydrated, and embedded in paraplast. Transverse sections $(7 \mu \mathrm{m})$ were stained with Goldner`s trichrome stain.

In Xenopus laevis Daudin several extrahepatic bile ducts leave right, median and left lobes of the liver and guide bile either via the cystic duct for storage into the gallbladder or via the common bile duct into the duodenum (Fig. 1). Ducts are supplied by branches of the hepatic artery (Fig. 1). Locally, ducts run side by side over varying distances before they merge to form a larger duct (Fig. 2). Supplying arteries give off first branches in the subserosa where a vascular plexus is formed (Fig. 3). Terminal branches finally capillarize subepithelially and form a dense capillary network (Fig. 4). Postcapillary venules run obliquely through the subepithelial lamina propria and finally drain into nearby venules (Fig. 4). Fig. 5 shows the junction of a larger extrahepatic duct and the cystic duct and their continuation as common bile duct. On its way to the duodenum the common bile duct receives further bile ducts coming from the median and the left lobes of the liver (Fig. 1). At the site where the common bile duct joins the duodenum the serosal vascular network of the common bile duct is continous with that of the duodenum (Fig. 6). By its architecture the extrahepatic bile duct microvasculature closely resembles that in mammals and thus it is considered to serve similar functions as that in mammals.

\section{References}

[1] S.H. Aharinejad and A. Lametschwandtner, J. Anat. 181 (1992) 89 
[2] O. Ohtani et al., Microsc. Res. Tech. 38 (1997) 660

[3] A. Caggiati et al., J. Anat. 180 (1992) 275

[4] H. Jackowiak and A. Lametschwandtner, Anat. Rec. 286A (2005) 974

[5] H. Jackowiak, Thesis for Habilitation. Rozprawy Naukowe 380, Poznan (Poland) 2006. ISSN 1896-1894.

[6] H. Jackowiak, B.Ciesielczyk and S. Godynicki. Papers of Symposium of Electron Microscopy, p 21-22. Rydzyna 2006.

[7] P. Häring, Thesis for Dr.of Dental Science (DDS).University of Basle, Switzerland 2006.

[8] This work was supported by a grant from the Fonds zur Förderung der Wissenschaftlichen Forschung, Project P-19050. The assistance of S. Tholo and Dr. W.D. Krautgartner is thankfully acknowledged.
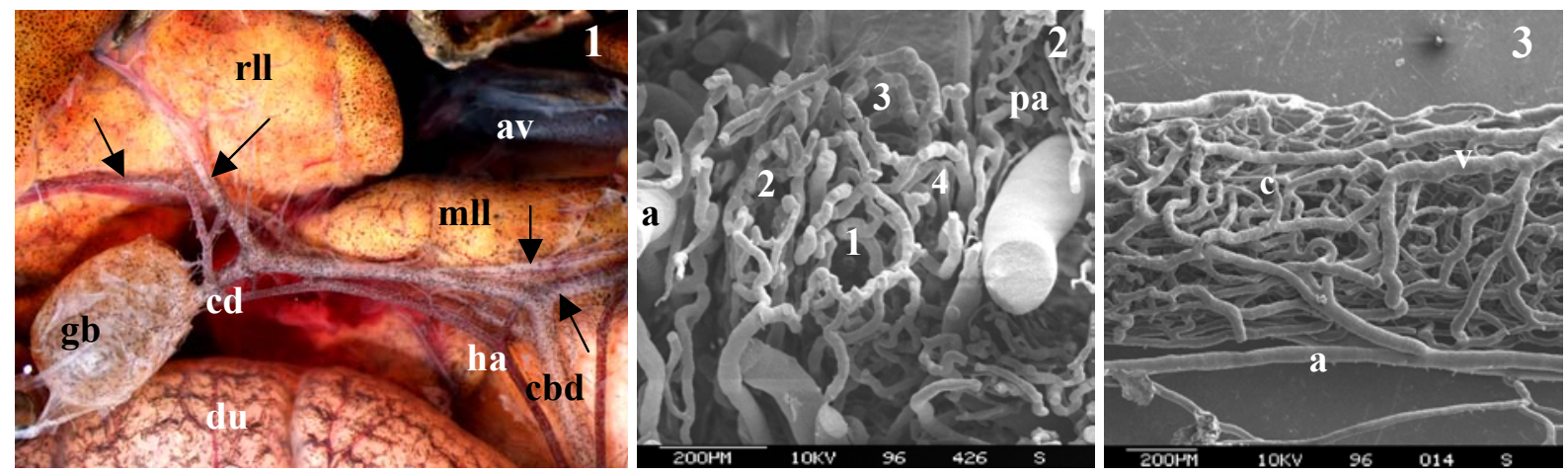

Fig. 1. Extrahepatic bile ducts (arrows) and gallbladder (gb) in adult Xenopus. Dissecting microscope. Ventral view. Right and left lobes of liver are flipped anteriorly. av abdominal vein. cbd common bile duct, cd cystic duct, du duodenum, ha hepatic artery, mll median lobe of liver, rll right lobe of liver. Anterior is on top. Fig. 2. Microvasculature of a bundle of extrahepatic bile ducts (1-4). VCC. Transverse section. a artery, pa pancreas. Fig. 3. Microvascular anatomy of the common bile duct. Serosal view. Vascular corrosion cast (VCC). SEM. a artery, c capillary, v vein.
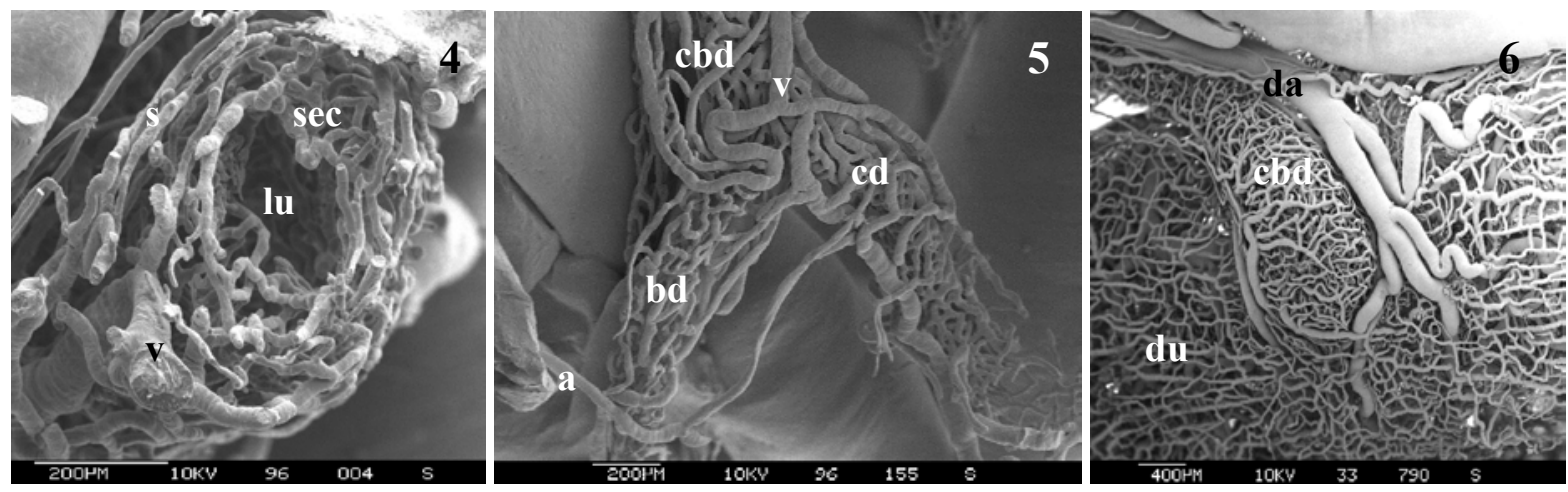

Fig. 4. Common bile duct. Transverse section. VCC. lu lumen, s serosal layer, sec subepithelial capillary network.v vein. Fig. 5. Extrahepatic bile duct (bd) from right lobe of liver, cystic duct (cd) and common bile duct (cbd). VCC. SEM. a artery, v vein. Fig. 6 Common bile duct (cbd) emptying into the duodenum (du). Serosal view. VCC. SEM. da duodenal artery. 